\title{
Role of lung ultrasound for the etiological diagnosis of acute lower respiratory tract infection (ALRTI) in children: a prospective study
}

\author{
Danilo Buonsenso ${ }^{1,2,3}$ (1) - Annamaria Musolino ${ }^{4} \cdot$ Valentina Ferro $^{4} \cdot$ Cristina De Rose $^{1} \cdot$ Rosa Morello $^{1}$. \\ Chiara Ventola ${ }^{1} \cdot$ Flora Marzia Liotti ${ }^{2} \cdot$ Rita De Sanctis $^{1} \cdot$ Antonio Chiaretti $^{1} \cdot$ Daniele Guerino Biasucci ${ }^{5}$. \\ Teresa Spanu ${ }^{2,6} \cdot$ Maurizio Sanguinetti $^{2,6} \cdot$ Piero Valentini $^{1}$
}

Received: 6 April 2021 / Accepted: 29 May 2021 / Published online: 19 June 2021

(c) The Author(s) 2021

\begin{abstract}
Objective and design Our prospective study assesses the role of detailed lung ultrasound (LUS) features to discriminate the etiological diagnosis of acute lower respiratory tract infection (ALRTI) in children.

Methodology We analyzed patients aged from 1 month to 17 years admitted between March 2018 and April 2020 who were hospitalized for ALRTI. For all patients, history, clinical parameters, microbiological data, and lung ultrasound data were collected. Patients were stratified into three main groups ("bacterial", "viral", "atypical") according to the presumed microbial etiology and LUS findings evaluated according to the etiological group. Nasopharyngeal swabs were obtained from all patients. A qualitative diagnostic test developed by Nurex S.r.l. was used for identification of bacterial and fungal DNA in respiratory samples. The Seegene Allplex ${ }^{\mathrm{TM}}$ Respiratory assays were used for the molecular diagnosis of viral respiratory pathogens. In addition, bacterial culture of blood and respiratory samples were performed, when indicated.

Results A total of 186 children with suspected ALRTI (44\% female) with an average age of 6 were enrolled in the study. We found that some ultrasound findings as size, number and distribution of consolidations, the position and motion of air bronchograms, pleural effusions and distribution of vertical artifacts significantly differ $(p<0.05)$ in children with bacterial, viral and atypical ALRTI.

Conclusion Our study provides a detailed analysis of LUS features able to predict the ALRTI ethology in children. These findings may help the physicians to better manage a child with ALRTI and to offer personalized approach, from diagnosis to treatment and follow-up.
\end{abstract}

Keywords Lung ultrasound · Pneumonia $\cdot$ ALRTI $\cdot$ Etiological diagnosis $\cdot$ Children $\cdot$ Personalized medicine

Danilo Buonsenso

danilobuonsenso@gmail.com

1 Department of Woman and Child Health and Public Health, Fondazione Policlinico Universitario A. Gemelli, Largo A. Gemelli 8, 00168 Rome, Italy

2 Dipartimento di Scienze Biotecnologiche di Base, Cliniche Intensivologiche e Perioperatorie, Università Cattolica del Sacro Cuore, Rome, Italy

3 Global Health Research Institute, Istituto di Igiene, Università Cattolica del Sacro Cuore, Roma, Italia

4 Department of Pediatric Emergency Medicine, Bambino Gesù Children's Hospital, IRCCS, Rome, Italy

5 Department of Anesthesia and Intensive Care, Fondazione Policlinico Universitario “A.Gemelli”, Rome, Italy

6 Dipartimento di Scienze di Laboratorio e Infettivologiche, Fondazione Policlinico Universitario A. Gemelli IRCCS, Rome, Italy

Abbreviations
$\begin{array}{ll}\text { ALRTI(s) } & \text { Acute Lower Respiratory Tract Infection(s) } \\ \text { LRTI } & \text { Lower Respiratory Tract Infection(s) } \\ \text { CXR } & \text { Chest X-ray } \\ \text { LUS } & \text { Lung ultrasound } \\ \text { CRP } & \text { C-reactive protein } \\ \text { ED } & \text { Emergency Department } \\ \text { WCC } & \text { White cell count } \\ \text { PCT } & \text { Procalcitonin } \\ \text { CT } & \text { Computed tomography }\end{array}$

\section{Introduction}

Acute lower respiratory tract infection (ALRTI) represents the single largest cause of pediatric morbidity and mortality worldwide $[1,2]$. The diagnosis of pneumonia is 
essentially based on medical history and clinical examination, which according to some studies has a poor sensitivity and specificity $[3,4]$. However, it do not allow to identify the causative agent responsible for the infection [5].

Although viruses represent the most frequent cause of ALRTI, the majority of children with suspected or confirmed ALRTI are still treated with empirical and often unnecessary antibiotics [6], contributing to the spread of antibiotic resistance, one of the biggest medical emergencies of modern medicine. In the Centers for Disease Control and Prevention Etiology of Pneumonia in the Community cohort, only $15 \%$ of hospitalized children with radiographic pneumonia had a detectable bacterial etiology; however, $88 \%$ received antibiotics [7]. A recently published among Pakistani children younger than 5 years of age with pneumonia with tachypnea randomized at amoxicillin vs placebo, the number of children with pneumonia and tachypnea who would have needed to be treated with amoxicillin to prevent one treatment failure was 44 , suggesting that a significant number of ALRTI is of viral origin and does not requires antibiotic [8].

However, current guidelines do not help the physician on how to approach to an optimized strategy for prevention, diagnosis and treatment of disease for each single child with ALRTI, based on his or her unique characteristics, but mainly suggest a general approach to pediatric ALRTI [9].

To date, according to some studies, both clinical findings [10] and laboratory results [10-16] could not entirely accurately distinguish viral, bacterial and atypical pneumonia. Furthermore, some studies, that report differences in laboratory biomarkers, could not determine reliable thresholds for differentiating bacterial pneumonia from viral pneumonia [13], since normal tests do not always exclude bacterial ALRTI [9].

Chest X-ray (CXR) is not necessary to confirm the diagnosis of ALRTI in milder cases, who are treated as outpatients and is also associated with risk of radiation exposure [17]. Moreover, CXR cannot reliably establish the microbial diagnosis of ALRTI [18-22] and the interpretation of radiographic images varies significantly among the observers [23].

In recent years, lung ultrasound (LUS) use has been widely studied as an alternative diagnostic tool for ALRTI of both bacterial and viral origin, proving to have high specificity and sensitivity for the diagnosis and as regards, the follow-up in children with pneumonia [21, 24-26]. Moreover, LUS has several advantages over X-ray, particularly useful for the pediatric population: radiation-free, lower cost, possibility of follow-up examinations, ability to monitor treatment, easy accessibility in all settings (including poor countries), fast, and can be used immediately as a point-ofcare method. LUS results are immediately available to the clinician, who must decide about the initial empirical treatment [19, 21-26].

The new challenge of LUS is to determine the etiology of ALRTI in the additional context of clinical and laboratory data. First studies primarily aimed to assess the role of LUS in ALRTI suggested that smaller consolidations may be associated with a viral etiology [27], but only one study prospectively assessed the role of LUS in defining the etiology of ALRTI [19]. However, although this study found that small subpleural consolidations and/or an increased number of B-lines (interstitial syndrome) are characteristics of viral pneumonia, the authors did not assessed important LUS features (such as the bronchograms, type and location of vertical artifacts, type of effusions).

Therefore, due to the growing role of LUS in the evaluation of patients with respiratory conditions and the limits of available data, we carried out this prospective study aiming to assess the role of LUS in supporting the etiological diagnosis of ALRTI in children.

\section{Participants and methods}

\section{Study population}

We prospectively analyzed patients aged from 1 month to 17 years admitted between March 2018 and April 2020 who were hospitalized for ALRTI and had pneumonic infiltrates detected with LUS. For all patients included in the study, history, clinical parameters, microbiological data, and ultrasound data were collected. The study was approved by the Ethics Committee of Fondazione Policlinic Universitario A. Gemelli IRCCS, Rome, Italy. Informed consent was obtained by all participants and/or their legal guardians (for children younger than 16 years). There is no identifying information or image in the article.

\section{Patients}

The evaluating physician made the clinical diagnosis of ALRTI in accordance with the British Thoracic Society guidelines [18]. Children with ALRTI may present with fever, tachypnea, breathlessness or difficulty in breathing, cough, wheeze or chest pain. They may also present with abdominal pain and/or vomiting and may have headache. These clinical features of pneumonia vary with the age of the child. In children older than 3 years, a history of difficulty breathing is an additional valuable symptom. A raised respiratory rate is associated with hypoxemia [18].

The recommendation of the guideline development group relevant to pneumonia is that chest radiography should not be considered a routine investigation in children thought to have ALRTI. Children with signs and symptoms of 
pneumonia who are not admitted to hospital should not have a chest $\mathrm{x}$-ray. Furthermore, according to the above guidelines, acute phase reactants are not of clinical utility in distinguishing viral from bacterial infections and should not routinely be tested and C-reactive Protein (CRP) is not useful in the management of uncomplicated pneumonia [18].

At the first evaluation in the Emergency Department (ED), all children with suspected ALRTI underwent medical history and clinical evaluation. Further investigations were performed only when deemed necessary from the evaluating pediatricians (anteroposterior CXR; blood tests including white cell count (WCC), C-reactive protein (CRP) and procalcitonin (PCT). The physician on duty-made decisions about the patient's diagnosis and treatment according to his/ her own practice and without knowledge of the LUS findings, but aware of other clinical/laboratory/imaging data if performed. In our institution, the local protocol for ALRTI antibiotic treatment follows the Pediatric Infectious Diseases Society and the Infectious Diseases Society of America [9].

\section{ALRTI definitions}

We defined as ALRTI those patients requiring: acute respiratory signs and symptoms, fever $>37.5^{\circ} \mathrm{C}$, clinical or radiological evidence of a new pulmonary infiltrates.

\section{Inclusion criteria}

Children with a clinical diagnosis of ALRTI (based on history, clinical examination, blood tests (if performed), and CXR (if performed) who underwent LUS within six hours from the first clinical evaluation and with available clinical information about the outcome (including the etiological suspicion) available.

\section{Exclusion criteria}

Patients with underlying diseases, including respiratory tract anomalies, immunodeficiency, cerebral palsy, neuromuscular diseases, congenital heart disease, and malignancy were excluded.

\section{Etiological stratifications of patients}

Patients were stratified into different groups according to the presumed microbial etiology: patients with bacterial pneumonia, patients with viral pneumonia, and patients positive for atypical pneumonia, especially Mycoplasma pneumoniae. An expert in pediatric infectious diseases assessed the final database, blinded to the clinical discharge charts, and classified the etiological diagnosis according to the following data.
Bacterial pneumonia was considered in patients documented bacterial infection (either culture- or PCR-based methods) in clinically significant samples (bronchoalveolar lavage, pleural drainage, blood cultures), and/or lobar pneumonia on CXR (if performed), and/or leucocytosis $(>15 \times 109 / \mathrm{L})$, and/or raised inflammatory markers (either CRP or PCT, according to clinical decision and local availability), even when viruses were detected in the nasopharyngeal swab. When no viruses or atypical bacteria were detected, the clinical opinion of the responsible pediatrician, based on clinical, laboratory and imaging studies, was considered enough for the stratification into the bacterial pneumonia group.

Patients with detected viral infection on nasopharyngeal were included into the viral pneumonia group only after the exclusion of bacterial superinfection, according to a comprehensive assessment of clinical, laboratory, radiology, and microbiological finding. Children were included into the atypical pneumonia group when Mycoplasma pneumoniae or Chlamydia pneumoniae were detected on nasopharyngeal swabs and bacterial superinfection was excluded, and clinical data were compatible with a diagnosis of atypical pneumonia.

\section{Microbiological studies}

Nasopharyngeal swabs were obtained and tested fresh from all patients. MicrobScan, a qualitative diagnostic test developed by Nurex S.r.l. (Sassari, Italy) was used for the detection and identification of bacterial and fungal DNA in respiratory samples. The target species panel includes Bordetella pertussis, Bordetella parapertussis, Legionella pneumophila, Chlamydia pneumoniae, Mycoplasma pneumonia, and the fungus Pneumocystis jirovecii. The Seegene Allplex ${ }^{\mathrm{TM}}$ Respiratory Panel 1, 2, and 3 assays were used for the molecular diagnosis of viral respiratory pathogens. The organisms detected by the three panels include influenza virus A (subtypes $\mathrm{H} 1$, pdm09 and $\mathrm{H} 3$ ), influenza virus $\mathrm{B}$, respiratory syncytial virus types $\mathrm{A}$ and $\mathrm{B}$, adenovirus, metapneumovirus, human enterovirus, parainfluenza virus 1 , parainfluenza virus 2 , parainfluenza virus 3 , parainfluenza virus. human bocavirus, coronavirus OC43, coronavirus 229E coronavirus, NL63 and human rhinovirus.

In addition, bacterial culture of blood and respiratory samples were performed, when indicated.

\section{Lung ultrasound}

LUS was performed with the ultrasound machine ESAOTE MyLab TM 40, which complies with the Medical Device Directive (MDD) 93\42 \EEC and subsequent amendments. In accordance with this directive, Esaote has classified it as Class IIa devices. It was performed within $6 \mathrm{~h}$ from the 
clinical diagnosis of ALRTI, unaware of microbiological, laboratory, and CXR (when performed) results.

Linear probe (12-6 MHz) was used in preschool children. In older children, we used a curved probe $(8-5 \mathrm{MHz})$.

Images and clips were stored and archived. All LUS was made by the same physician that made the first one to reduce inter-operator differences.

The following LUS features were recorded (Fig. 1):
- Size of the main lesion, that we generally define as subpleural lung parenchymal lesion (consolidation and Atelectasis), if they are single or multiple and location (monolateral or bilateral)

- Presence of bronchograms, its characteristics (air or fluid), morphology (arboriform or dot-like/linear), position (deep if $>2 \mathrm{~cm}$ far from the pleura or superficial if close to the pleura), dynamicity during breath (fix, poorly dynamic, or clearly dynamic);
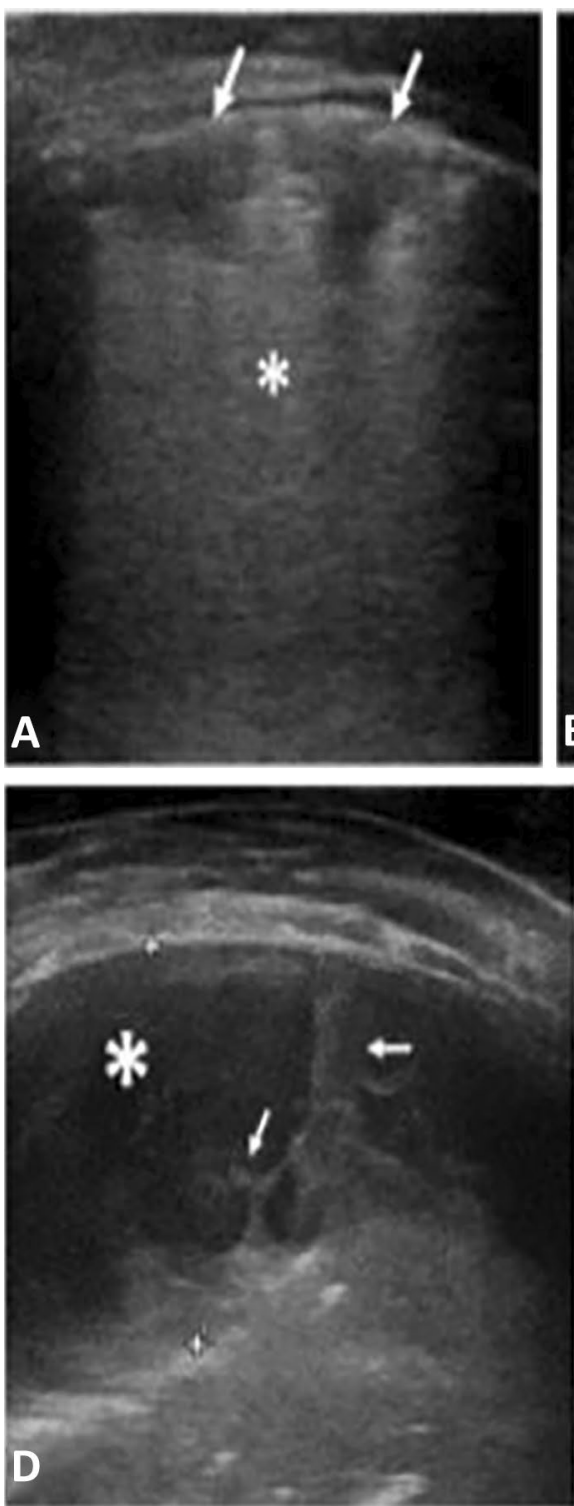
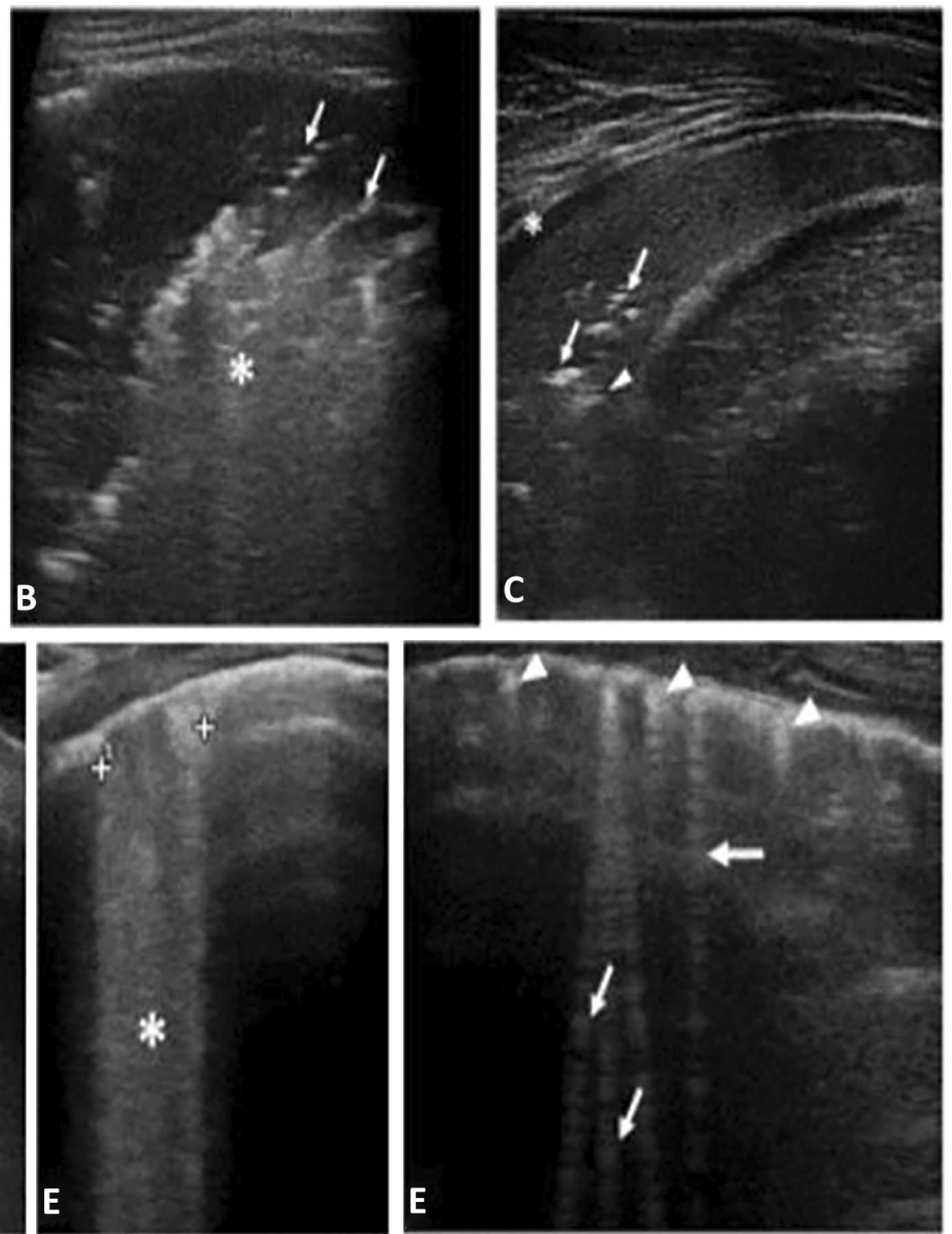

Fig. 1 A. Viral Pneumonia (H1N1): sub-centimeter subpleural consolidation (arrows) associated with vertical long perilesional vertical artifacts and areas of white lung (asterisk). B Atypical Pneumonia (Mycoplasma pneumoniae): Subpleural consolidation of less than $4 \mathrm{~cm}$ in size with dynamic superficial air bronchogram (arrow) and perilesional and deep confluent vertical artifacts (asterisk). C. BACTERIAL PNEUMONIA: Compact/hepatized large subpleural consol- idation with static air bronchograms (arrow) and deep fluid bronchogram (arrowhead). Minimum reactive pleural effusion (asterisk). D Complex Pleural Effusion in a bacterial Pneumonia: complicated pleural effusion (asterisk) with multiple and concamerated fibrin (arrow). E Confluent long vertical artifacts (asterisk). F Isolated long vertical artifacts (arrow); short vertical artifacts (arrowhead) 
- Presence of vertical artifacts or B lines, charactheristics (short or long, spared or confluents), position (monolateral or bilateral, perilesional or not)

- Presence and type of pleural effusion: simple (anechogenic and dependent to gravity) or complex (presence of septa, hyperechogenic spot, following the lung through the apex and not dependent to gravity, requiring drainage).

The scans were made by investigating the anterior, lateral and posterior regions of the thorax and placing the probe transversally and longitudinally along the lines considered traditional ultrasound findings: the parasternal line, the axillary line and the paravertebral line so as to fully explore the chest wall according to a methodical scheme first described by Copetti et al. [24]. To investigate the anterior and lateral lung fields patients were positioned, according to age, in a seated or supine position. The posterior lung fields have been explored in lateral decubitus and in sitting position.

We compared the ultrasound results with the microbiological tests performed to verify the concordance of the etiological diagnosis, according to the three main groups "bacterial", "viral", "atypical".

\section{Treatment}

We considered standard of care (SOC) the first line treatments (for either outpatient or inpatient) as per international guidelines [9] (amoxicillin, amoxicillin/clavulanate, ceftriaxone, cefotaxime, ampicillin, and penicillin).

\section{Statistical analysis}

To determine the power of the study, the study was set up as a study of agreement between lung ultrasound and the etiology of ALRTI. For atypical pneumonia, we considered a $60 \%$ agreement (then a $40 \%$ disagreement); for bacterial pneumonia, an agreement of $90 \%$ (disagreement $10 \%$ ). To be conservative, we considered the highest percentage of disagreement that is $40 \%$. In this case considering alpha $=0.01$ and beta $=0.20, n=159$. A Statistical analysis was performed using the software STATA/IC 14.2 version 2017. We use the Skewness/Kurtosis test to verify the normality of the distribution. Continuous variables showed non-parametric distribution and were presented as median [interquartile range (IQR)]. Categorical variables were reported as frequency and percentage. Categorical variables were compared using chi-squared or Fisher's exact test, as appropriate. Continuous variables were assessed using Mann-Whitney $U$ test. We performed a multivariable logistic regression analysis to study the relationship between the etiological diagnosis of the ALRTI as the dependent variable and lung US such as lung consolidation, small subpleural consolidation, B lines, confluent lines) as the independent variables. Inclusion of variables in the model was based on clinical plausibility and significant differences on $\chi^{2}$ and Mann-Whitney $U$ test. The model was adjusted for age, sex, clinical and laboratory characteristics. Variables with extremely unbalanced distribution in the two groups (frequency $0 \%$ in one groups) were excluded. We considered a two-tailed $p$ value less than 0.05 to be significant. The dataset is available upon reasonable request. The interrater reliability for each variable was with the Cohen's $k$ coefficient. The concordance was considered absent for $k$ values lower than 0 , poor if between 0 and 0.4 ; discrete if between 0.4 and 0.6 , good if $k$ between 0.6 and 0.8 ; high if above 0.8

\section{Results}

\section{Patients' characteristics}

A total of 186 children with suspected ALRTI (103 male and 83 female) with an average age of six were enrolled in the study (Fig. 2). The prevalent etiology of ALRTI was viral $(40.86 \%)$, with a slight prevalence in male (55\% of the cases). Main epidemiological and clinical characteristics of the study population, assessed according to the etiological group, are described in detail in Table 1. As reported, the majority of clinical parameters were not able to discriminate the different etiological groups.

Main diagnostic and laboratory investigations, assessed according to the etiological group, are described in detail in Table 2. Neither laboratory nor CXR findings were able to significantly discriminate the etiological groups since, also in case of statistical significance, the overlap between etiological groups was evident.

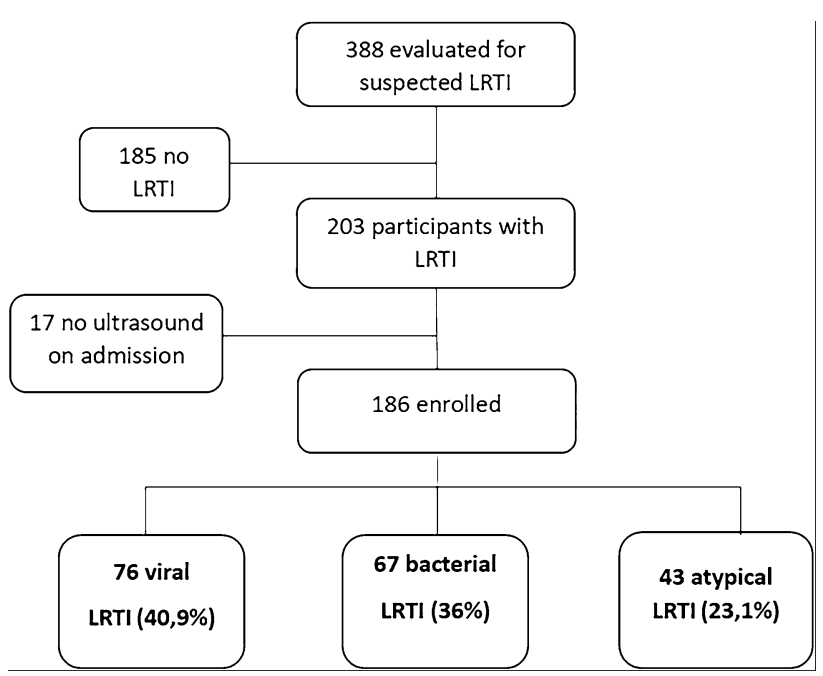

Fig. 2 Study flow 
Table 1 Differences of the epidemiological and clinical features in community acquired pneumonia with three etiological causative agents

\begin{tabular}{|c|c|c|c|c|}
\hline Epidemiological/clinical characteristics & $\begin{array}{l}\text { Bacterial infection } \\
n=67\end{array}$ & $\begin{array}{l}\text { Viral infection } \\
n=76\end{array}$ & $\begin{array}{l}\text { Atypical infection } \\
n=43\end{array}$ & $P$ value \\
\hline \multicolumn{5}{|l|}{ Gender } \\
\hline Female & $27(40.30)$ & $34(44.74)$ & $22(51.16)$ & B-V: 0.592 \\
\hline Male & $40(59.70)$ & $42(55.26)$ & $21(48.84)$ & $\begin{array}{l}\text { V-A: } 0.5 \\
\text { B-A:0.263 }\end{array}$ \\
\hline Age (years, median, SD) & $4(7)$ & $2(5.5)$ & $7(5)$ & $\begin{array}{l}\text { B-V: } 0.011 \\
\text { V-A: }<0.001 \\
\text { B-A: } 0.002\end{array}$ \\
\hline Fever & $56(83.54)$ & $61(80.26)$ & $32(74.42)$ & $\begin{array}{l}\text { B-V: } 0.608 \\
\text { V-A: } 0.459 \\
\text { B-A: } 0.241\end{array}$ \\
\hline Cough & $40(59.70)$ & $51(67.11)$ & $37(86.05)$ & $\begin{array}{l}\text { B-V: } 0.358 \\
\text { V-A: 0.024 } \\
\text { B-A:0.003 }\end{array}$ \\
\hline Chest pain & $10(14.93)$ & $4(5.26)$ & $4(9.30)$ & $\begin{array}{l}\text { B-V: } 0.05 \\
\text { V-A:0.398 } \\
\text { B-A:0.282 }\end{array}$ \\
\hline Respiratory distress & $19(28.79)$ & $20(26.67)$ & $3(6.98)$ & $\begin{array}{l}\text { B-V: } 0.779 \\
\text { V-A:0.007 } \\
\text { B-A:0.004 }\end{array}$ \\
\hline Wheezing (at auscultation) & $16(25)$ & $27(36)$ & $11(25.54)$ & $\begin{array}{l}\text { B-V:0.162 } \\
\text { V-A:0.224 } \\
\text { B-A:0.94 }\end{array}$ \\
\hline Crackles (at auscultation) & $26(40.63)$ & $21(28.38)$ & $20(46.51)$ & $\begin{array}{l}\text { B-V: } 0.130 \\
\text { V-A:0.047 } \\
\text { B-A:0.546 }\end{array}$ \\
\hline Decreased air entry (at auscultation) & $46(71.88)$ & $34(45.95)$ & $23(53.49)$ & $\begin{array}{l}\text { B-V: } 0.002 \\
\text { V-A:0.431 } \\
\text { B-A:0.05 }\end{array}$ \\
\hline Need oxygen (low flow) & $19(28.36)$ & $21(27.63)$ & $3(6.98)$ & $\begin{array}{l}\text { B-V: } 0.9 \\
\text { V-A:0.005 } \\
\text { B-A: } \mathbf{0 . 0 0 5}\end{array}$ \\
\hline Need oxygen (HNFC) & $12(17.21)$ & $18(23.68)$ & $8(18.60)$ & $\begin{array}{l}\text { B-V: } 0.4 \\
\text { V-A: } 0.519 \\
\text { B-A:0.927 }\end{array}$ \\
\hline Need oxygen (CPAP) & $4(5.97)$ & $4(5.96)$ & $1(2.33)$ & $\begin{array}{l}\text { B-V: } 0.85 \\
\text { V-A:0.403 } \\
\text { B-A:0.348 }\end{array}$ \\
\hline Need intubation & $8(11.94)$ & $2(2.63)$ & 0 & $\begin{array}{l}\text { B-V: } \mathbf{0 . 0 3 1} \\
\text { V-A: } 0.406 \\
\text { B-A: } \mathbf{0 . 0 1 6}\end{array}$ \\
\hline Need intensive care & $4(6.06)$ & $1(1.32)$ & 0 & $\begin{array}{l}\text { B-V:0.142 } \\
\text { V-A:0.639 } \\
\text { B-A: } 0.130\end{array}$ \\
\hline Complicated clinical course & $26(38.81)$ & $6(7.89)$ & $1(2.33)$ & $\begin{array}{l}\text { B-V: }<\mathbf{0 . 0 0 1} \\
\text { V-A: } 0.207 \\
\text { B-A: }<\mathbf{0 . 0 0 1}\end{array}$ \\
\hline Medical disposition hospitalization & $64(95.52)$ & $65(85.53)$ & $32(74.44)$ & $\begin{array}{l}\text { B-V: } \mathbf{0 . 0 4 5} \\
\text { V-A:0.134 } \\
\text { B-A:0.001 }\end{array}$ \\
\hline Length of hospitalization (days) & $8(12)$ & $5(4)$ & $3(5)$ & $\begin{array}{l}\text { B-V: }<0.001 \\
\text { V-A: } 0.0025 \\
\text { B-A: }<0.001\end{array}$ \\
\hline Length of therapy (days) & $10(10)$ & $7(5)$ & $7(5)$ & $\begin{array}{l}\text { B-V }<0.001 \\
\text { V-B: } 0.07 \\
\text { B-A: }<0.001\end{array}$ \\
\hline
\end{tabular}


Table 2 Diagnostic/laboratory investigations

\begin{tabular}{|c|c|c|c|c|}
\hline Diagnostic/laboratory investigations & $\begin{array}{l}\text { Bacterial infection } \\
n=67\end{array}$ & $\begin{array}{l}\text { Viral infection } \\
n=76\end{array}$ & $\begin{array}{l}\text { Atypical infection } \\
n=43\end{array}$ & $p$ value \\
\hline Performed chest X-ray (CXR) & $64(95.52)$ & $63(84)$ & $33(76.74)$ & $\begin{array}{l}\text { B-V: } 0.026 \\
\text { V-A:0.330 } \\
\text { B-A:0.003 }\end{array}$ \\
\hline Consolidation (CXR) & $58(90.63)$ & 49 (77.78) & $27(81.82)$ & $\begin{array}{l}\text { B-V: } 0.047 \\
\text { V-A: } 0.643 \\
\text { B-A:0.212 }\end{array}$ \\
\hline Atelectasis (CXR) & $12(18.75)$ & $12(19.05)$ & $3(9.09)$ & $\begin{array}{l}\text { B-V: } 0.9 \\
\text { V-A:0.164 } \\
\text { B-A:0.172 }\end{array}$ \\
\hline Pleural effusion (CXR) & $39(60.94)$ & $15(23.81)$ & $9(27.27)$ & $\begin{array}{l}\text { B-V: }<\mathbf{0 . 0 0 1} \\
\text { V-A:0.710 } \\
\text { B-A:0.002 }\end{array}$ \\
\hline $\mathrm{CRP}<50 \mathrm{mg} / \mathrm{L}$ & $24(40.68)$ & $52(85.25)$ & $15(65.22)$ & $\begin{array}{l}\text { B-V: }<\mathbf{0 . 0 0 1} \\
\text { V-A: } 0.042 \\
\text { B-A: } 0.046\end{array}$ \\
\hline CRP $50-100 \mathrm{mg} / \mathrm{L}$ & $12(20.34)$ & $3(4.92)$ & $3(13.04)$ & $\begin{array}{l}\text { B-V:0.01 } \\
\text { V-A: } 0.202 \\
\text { B-A:0.336 }\end{array}$ \\
\hline $\mathrm{CRP}>100 \mathrm{mg} / \mathrm{L}$ & $23(38.98)$ & $6(9.84)$ & $5(21.74)$ & $\begin{array}{l}\text { B-V: }<\mathbf{0 . 0 0 1} \\
\text { V-A:0.141 } \\
\text { B-A:0.139 }\end{array}$ \\
\hline $\begin{array}{l}\text { WBC count: } \\
<10,000 / \mu \mathrm{L}\end{array}$ & $23(41.07)$ & $23(42.59)$ & $9(37.50)$ & $\begin{array}{l}\text { B-V:0.872 } \\
\text { V-A: } 0.673 \\
\text { B-A:0.766 }\end{array}$ \\
\hline $\begin{array}{l}\text { WBC count: } \\
10,000-15,000 / \mu \mathrm{L}\end{array}$ & 15 (26.79) & $18(33.33)$ & $8(33.33)$ & $\begin{array}{l}\text { B-V:0.454 } \\
\text { V-A:1 } \\
\text { B-A:0.553 }\end{array}$ \\
\hline $\begin{array}{l}\text { WBC count: } \\
>15,000 / \mu \mathrm{L}\end{array}$ & $18(32.14)$ & $13(24.07)$ & $7(29.17)$ & $\begin{array}{l}\text { B-V: } 0.347 \\
\text { V-A:0.635 } \\
\text { B-A:0.792 }\end{array}$ \\
\hline
\end{tabular}

\section{LUS findings}

Details about LUS features in children with bacterial, viral and atypical pneumonia are described in Table 3 and in Fig. 3 (Box Plot of Consolidation Size by Etiology). Consolidations were found in $100 \%$ of bacterial infections, with a significant statistical difference compared to viral infections $(p$ value $=0.001)$ and to atypical infections $(p$ value $=0.004)$. We analyzed the size of consolidation according to the etiological group:

- For consolidation $<1.5 \mathrm{~cm}$, there is a statistical significance between bacterial and viral infections ( $p$ value $=<0.001)$ and between bacterial and atypical infections $(p$ value $=0.004)$. They were more expressed in viral pneumonia (63.8\%);

- For consolidation between 1.5 and $4 \mathrm{~cm}$, there is a statistical significance between bacterial and viral infections ( $p$ 0.022), and they were more expressed in bacterial pneumonia $(55.22 \%)$;
- For consolidation $>4 \mathrm{~cm}$, there is a statistical significance between bacterial and viral infections $(p$ value $<0.001)$ and between bacterial and atypical infections ( $p$ value $<0.001$ ). They were more expressed in bacterial infections $(22.39 \%)$ and poorly expressed in viral infections (1.54\%).

Multiple and bilateral consolidation are infrequent in bacterial infections, making them statistically significant to distinguish bacterial pneumonia from viral and atypical diseases $(p<0.0001)$.

We also considered air bronchogram, that was highly represented in bacterial $(77.61 \%)$ and atypical $(60.47 \%)$ ALRTI. There is a statistical significant difference between bacterial infections and viral infections ( $p$ value $\leq 0.001$ ) and between viral infections and atypical infections ( $p$ value $=0.004)$. Different characteristics of the air bronchogram have been taken into consideration: 
Table 3 Differences of the ultrasound features in community acquired pneumonia with three etiological causative agents

\begin{tabular}{|c|c|c|c|c|}
\hline Characteristics lung US & $\begin{array}{l}\text { Bacterial infection } \\
n=67\end{array}$ & $\begin{array}{l}\text { Viral infection } \\
n=76\end{array}$ & $\begin{array}{l}\text { Atypical infection } \\
n=43\end{array}$ & $p$ value \\
\hline Consolidation & $67(100)$ & $65(85.53)$ & $38(88.37)$ & $\begin{array}{l}\text { B-V: } 0.001 \\
\text { V-A:0.66 } \\
\text { B-A: 0.004 }\end{array}$ \\
\hline \multicolumn{5}{|l|}{ Size consolidation } \\
\hline$<1.5 \mathrm{~cm}$ & $15(22.39)$ & $41(63.8)$ & $19(50)$ & $\begin{array}{l}\text { B-V: }<\mathbf{0 . 0 0 1} \\
\text { V-A: } 0.19 \\
\text { B-A: } \mathbf{0 . 0 0 4}\end{array}$ \\
\hline $1.5-4 \mathrm{~cm}$ & $37(55.22)$ & $23(35.38)$ & $17(44.74)$ & $\begin{array}{l}\text { B-V: } \mathbf{0 . 0 2 2} \\
\text { V-A: } 0.347 \\
\text { B-A: } 0.301\end{array}$ \\
\hline$>4 \mathrm{~cm}$ & $15(22.39)$ & $1(1.54)$ & $2(5.26)$ & $\begin{array}{l}\text { B-V: }<\mathbf{0 . 0 0 1} \\
\text { V-A: } 0.306 \\
\text { B-A:0.022 }\end{array}$ \\
\hline Multiple consolidations & $5(7.46)$ & $28(43.8)$ & $13(34.21)$ & $\begin{array}{l}\text { B-V: }<\mathbf{0 . 0 0 1} \\
\text { V-A: } 0.375 \\
\text { B-A: }<\mathbf{0 . 0 0 1}\end{array}$ \\
\hline Bilateral consolidations & $6(0.09)$ & $31(46.15)$ & $12(31.58)$ & $\begin{array}{l}\text { B-V: }<\mathbf{0 . 0 0 1} \\
\text { V-A: } 0.146 \\
\text { B-A:0.004 }\end{array}$ \\
\hline Air bronchogram & $52(77.61)$ & $25(32.89)$ & $26(60.47)$ & $\begin{array}{l}\text { B-V: }<\mathbf{0 . 0 0 1} \\
\text { V-A:0.004 } \\
\text { B-A:0.053 }\end{array}$ \\
\hline \multicolumn{5}{|l|}{ Position air bronchogram } \\
\hline Deep & $28(53.85)$ & $14(56)$ & $6(23.08)$ & B-V: 0.859 \\
\hline Superficial & $24(46.15)$ & $11(44)$ & $20(76.92)$ & $\begin{array}{l}\text { V-A:0.016 } \\
\text { B-A: } 0.010\end{array}$ \\
\hline \multicolumn{5}{|l|}{ Bronchogram } \\
\hline Dynamic & $22(44)$ & $9(37.50)$ & $18(69.23)$ & B-V: 0.596 \\
\hline Static & $28(56)$ & $15(62.50)$ & $8(30.77)$ & $\begin{array}{l}\text { V-A:0.025 } \\
\text { B-A:0.037 }\end{array}$ \\
\hline Fluid bronchogram & $22(32.84)$ & $3(3.95)$ & $4(9.30)$ & $\begin{array}{l}\text { B-V: }<\mathbf{0 . 0 0 1} \\
\text { V-A:0.233 } \\
\text { B-A:0.003 }\end{array}$ \\
\hline Pleural effusion & $33(49.25)$ & $12(15.79)$ & $7(16.28)$ & $\begin{array}{l}\text { B-V: }<\mathbf{0 . 0 0 1} \\
\text { V-A:0.94 } \\
\text { B-A: }<\mathbf{0 . 0 0 1}\end{array}$ \\
\hline Complicated effusion & $3(10.34)$ & 0 & $2(33.33)$ & $\begin{array}{l}\text { B-V: } 0.374 \\
\text { V-A: } 0.192 \\
\text { BA: } 0.143\end{array}$ \\
\hline Vertical deep artifacts & $39(58.21)$ & $67(88.16)$ & $28(88.37)$ & $\begin{array}{l}\text { B-V: }<\mathbf{0 . 0 0 1} \\
\text { V-A: } 0.972 \\
\text { B-A: } \mathbf{0 . 0 0 1}\end{array}$ \\
\hline \multicolumn{5}{|l|}{ Vertical deep artifacts } \\
\hline Diffuse & 17 (44.74)17 (44.74) & $55(78.57)$ & $26(70.27)$ & B-V: $<0.001$ \\
\hline Peri-lesion & $21(56.6)$ & $15(21.43)$ & $11(29.73)$ & $\begin{array}{l}\text { V-A:0.341 } \\
\text { B-A: } \mathbf{0 . 0 2 5}\end{array}$ \\
\hline \multicolumn{5}{|l|}{ Vertical deep artifacts } \\
\hline Confluent & $1(2.63)$ & $4(5.71)$ & 0 & $\begin{array}{l}\text { B-V: } 0.467 \\
\text { V-A:0.123 } \\
\text { B-A: } 0.5\end{array}$ \\
\hline
\end{tabular}

- Position: deep air bronchogram was found in $53.85 \%$ of bacterial pneumonia and $56 \%$ of viral pneumonia; superficial air bronchogram was almost always present in the atypical pneumonia (76.92\%). Position is a valid statistical parameter to distinguish atypical infection from the bacterial one $(p$ value $=0.010)$ and the viral one ( $p$ value $=0.010$ ); however, it is not a statistically 


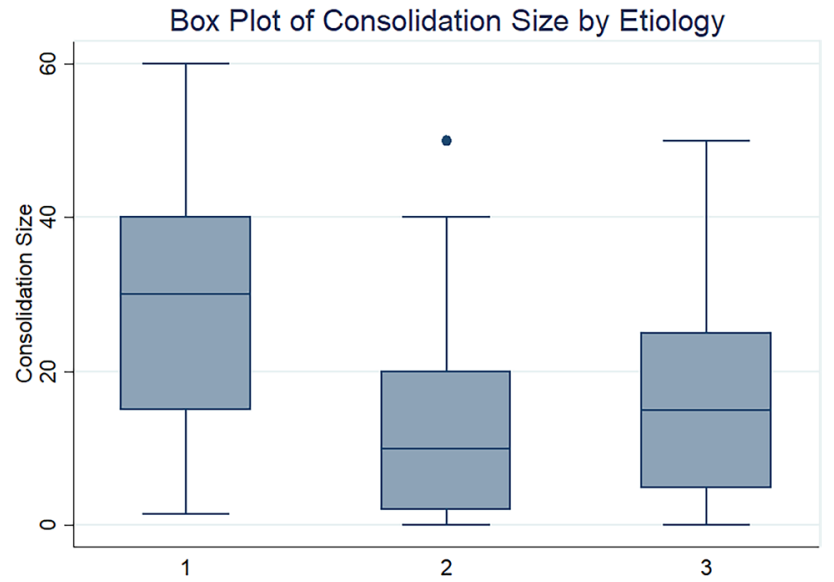

Fig. 3 Box plot of consolidation size by etiology

significant parameter for distinguish between the bacterial and viral pneumonia ( $p$ value $=0.859$ );

- Dynamic bronchogram was often found in atypical infections $(69.23 \%)$ and moderately represented in bacterial (44\%) and viral (37.50\%) infections. Static bronchogram were found in $56 \%$ of cases in bacterial forms, $62.50 \%$ of cases in viral forms and in $30.77 \%$ of cases in atypical forms $(p>0.05)$.

- The presence of fluid bronchogram was more frequently described in case of bacterial pneumonia compared with viral $(p<0.001)$ and atypical pneumonia $(p 0.003)$.

Pleural effusions were significantly more frequent in children with bacterial ALRTI $(p<0.0001)$. Complicated effusion was not represented in viral forms.

We also assessed vertical deep artifacts. They were frequently found in the viral (88.16\%) and atypical (88.37\%) infections, and moderately found in the bacterial ones $(58.21 \%)$. Children with viral and atypical pneumonia had significantly more vertical deep artifacts compared with bacterial pneumonia ( $p<0.001$ and $p 0.001$, respectively), while there were no significant differences between viral and atypical pneumonia $(p>0.05)$. Also the distribution of the vertical deep artifacts in the three groups, being significantly more diffuse in children with viral $(78.57 \%)$ and atypical (70.27\%) ALRTI $(p<0.05)$, while children with bacterial ALRTI mainly had vertical artifacts located in continuity with the main consolidation.

Tables 4, 5 and 6 show the multivariable logistic regression analyses applied to compared the three main groups.

\section{Interobserver agreement}

We made interobserver agreement on a random set of $10 \%$ images. The levels of interobserver agreement were good (values of coefficient between 0.6 and 0.8 ) for air
Table 4 Logistic regression model analyzing the diagnostic (clinical, laboratory and US) predictors for bacterial infection versus viral infection in community acquired pneumonia

\begin{tabular}{lrlllr}
\hline B-V infection & \multicolumn{1}{c}{ OR } & \multicolumn{1}{l}{$p$} & \multicolumn{2}{c}{$95 \%(\mathrm{CI})$} \\
\hline Gender (female vs. male) & 0.75 & -0.46 & 0.643 & 0.22 & 2.57 \\
Age (years) & 1.23 & 2.43 & 0.015 & 1.04 & 1.44 \\
Large sized consolidation & 13.62 & 2.08 & 0.038 & 1.16 & 159.88 \\
Multiple consolidation & 0.24 & -1.42 & 0.154 & 0.03 & 1.72 \\
Bilateral consolidation & 0.23 & -1.43 & 0.152 & 0.03 & 1.72 \\
Air bronchogram & 6.58 & 2.69 & 0.007 & 1.67 & 25.93 \\
Deep vertical artifacts & 0.27 & -1.88 & 0.060 & 0.07 & 1.06 \\
Pleural effusion & 1.48 & 0.61 & 0.543 & 0.42 & 5.16 \\
CRP $>100$ mg/L & 15.94 & $3-43$ & 0.001 & 3.28 & 77.50 \\
Chest pain & 0.47 & -0.80 & 0.426 & 0.07 & 2.99 \\
Decreased air entry (ausculta- & 0.75 & -0.46 & 0.643 & 0.22 & 2.57 \\
$\quad$ tion) & & & & & \\
Constant & 0.30 & -1.12 & 0.261 & 0.04 & 2.46 \\
\hline
\end{tabular}

The model showed that the likelihood of the bacterial etiology increased significantly of more than 13 times for the large sized consolidation ( $p=0.039$; OR: 13.62; 95\% CI 1.16-159.88). The air bronchogram occurred more than six times in the bacterial community pneumonia. ( $p=0.007$; OR: $6.58 ; 95 \%$ CI $0.67-25.93$ ). The CRP value of $100 \mathrm{mg} / \mathrm{L}$ was about 15 times as much associated with bacterial etiology ( $p=0.001$; OR: 15.94; 95\% CI 3.28-77.50). In addition, the more age increased, the greater the likelihood of bacterial etiology ( $p=0.015$; OR: 1.23 ; 95\% CI 1.04-1.44)

$O R$ odds ratio, $z$ regression coefficient divided by its standard error, 96\% CI 95\% confidence interval

bronchogram position (deep, superficial) and motion (static, dynamic), while was above 0.8 (confirming a high interobserver agreement) for consolidation size, effusions, presence and distribution of vertical artifacts.

Table 5 Logistic regression model analyzing the diagnostic (clinical laboratory and US) predictors for viral infection versus atypical infection in community acquired pneumonia

\begin{tabular}{lrrllr}
\hline V-A & OR & \multicolumn{1}{l}{$Z$} & \multicolumn{3}{l}{$95 \%(\mathrm{CI})$} \\
\hline Gender (female vs. male) & 0.41 & -1.35 & 0.176 & 0.11 & 1.49 \\
Age (years) & 0.93 & -1.00 & 0.319 & 0.80 & 1.07 \\
Air bronchogram & 0.14 & -2.84 & 0.004 & 0.04 & 0.55 \\
Cough & 0.33 & -1.25 & 0.210 & 0.06 & 1.87 \\
Respiratory distress & 8.35 & 1.80 & 0.073 & 0.82 & 84.56 \\
Crackles (at auscultation) & 0.58 & -0.80 & 0.426 & 0.15 & 2.23 \\
CRP <50 mg/L & 4.15 & 1.74 & 0.082 & 0.84 & 20.66 \\
Constant & 13.27 & 2.47 & 0.014 & 1.70 & 103.56 \\
\hline
\end{tabular}

The model showed that the detection of air bronchogram at lung US reduced by $86 \%$ the odds of viral CAP ( $p=0.004$; OR: $0.14 ; 95 \% \mathrm{CI}$ $0.04-0.55)$

$O R$ Odds ratio $z$ regression coefficient divided by its standard error, 96\% CI 95\% confidence interval 
Table 6 Logistic regression model analyzing the diagnostic (clinical, laboratory and US) predictors for bacterial infection versus atypical infection in community acquired pneumonia

\begin{tabular}{|c|c|c|c|c|c|}
\hline B-A & OR & $z$ & $p$ & $95 \%$ & CI) \\
\hline Gender (female vs. male) & 0.36 & -1.04 & 0.30 & 0.05 & 2.48 \\
\hline Age (years) & 0.81 & -1.81 & 0.07 & 0.65 & 1.02 \\
\hline Small sized consolidation & 0.82 & -0.18 & 0.86 & 0.09 & 7.65 \\
\hline Air bronchogram & 0.41 & -0.72 & 0.47 & 0.04 & 4.57 \\
\hline Pleural effusion & 12.70 & 1.88 & 0.06 & 0.89 & 180.29 \\
\hline Bilateral consolidation & 0.26 & -0.59 & 0.56 & 0.00 & 24.26 \\
\hline Multiple consolidation & 0.36 & -0.56 & 0.57 & 0.01 & 12.72 \\
\hline Deep vertical artifacts & 1.00 & & & & \\
\hline $\mathrm{CRP}<50 \mathrm{mg} / \mathrm{dL}$ & 0.06 & -2.43 & 0.02 & 0.01 & 0.58 \\
\hline Cough & 0.27 & -0.85 & 0.40 & 0.01 & 5.46 \\
\hline Respiratory distress & 1.00 & & & & \\
\hline Decreased air entry & 0.53 & -0.61 & 0.54 & 0.07 & 4.10 \\
\hline Constant & 310.84 & 2.33 & 0.02 & 2.50 & $38,716.21$ \\
\hline
\end{tabular}

The model showed that the value of $\mathrm{CRP}<50 \mathrm{mg} / \mathrm{dL}$ reduced by $94 \%$ the odds of bacterial etiology compared with atypical etiology ( $p=0.02$; OR: 0.06 ; 95\% CI 0.01-0.58)

$O R$ odds ratio, $z$ regression coefficient divided by its standard error, $96 \%$ CI $95 \%$

\section{Discussion}

Our prospective study is one of the largest prospective studies assessing the role of detailed LUS features to discriminate bacterial, viral and atypical ALRTI in children. We found that the size, number and distribution of consolidations, the position and motion of air bronchograms, pleural effusions and distribution of vertical artifacts significantly differ in children with bacterial, viral and atypical ALRTI. Such differences were particularly relevant when bacterial ALRTIs were compared with viral and atypical ones. Conversely, clinical parameters, including fever, chest pain and main auscultation features, and laboratory were not able to significantly distinguish between these groups. CXR, despite being still widely used, was the less useful tool in this discrimination. These findings highlight, therefore, the need of using new tools to support the etiological classification of pediatric ALRTI. We proved that several LUS features may easily support clinicians in this using the LUS along with available clinical, anamnestic and laboratory data.

Given the increasing phenomenon of antibiotic resistance in children, the possibility of knowing the etiology of the infection represents an important step forward. The first decade of LUS studies focused on the role of LUS in detecting pneumonia. A recently performed meta-analysis confirmed high sensitivity (96\%) and specificity (93\%) of LUS for detecting pneumonia in children [4]. First studies showed specific LUS patterns to diagnose viral lower respiratory tract infections and bronchiolitis in children
[28-31]. Buonsenso and colleagues [15, 24] showed that specific LUS patterns on diagnosis and after $48 \mathrm{~h}$ of treatments (bronchograms, consolidation size, characteristics of pleural effusion) were predictive of antibiotic response in children with ALRTI, more than clinical data and laboratory results. Moreover, recent basic science and clinical studies are clarifying the knowledge of and genesis of artifacts generated by the ultrasound-lung interaction. In particular, performing physical analyses on artificial models and using modern deep learning strategies and train a fully convolutional neural network, Demi and colleagues [32-34] showed that B-lines have different morphologies according to the medical conditions that generate them (interstitial lung diseases, cardiogenic and non-cardiogenic lung edema, interstitial pneumonia and lung contusion).

The ultrasound analyzes (vertical artifacts, consolidations, echogenicity, bronchograms, and dynamic data) offered by our study represent a modern view of LUS which, in this way, may represent a new and promising tool to personalize the diagnosis, treatment, follow-up and care of children with ALRTI, as recently highlighted by international experts [35]: during the last decades, LUS moved from being a diagnostic tool (qualitative approach) to a monitoring tool for lung aeration quantification (ie, quantitative approach). Artificial intelligence softwares, since we are entering the era of the digital medicine (a process particularly speed-up by the COVID-19 pandemic), will also support this view [36].

To date, few studies have described LUS findings able to define the etiology of pediatric. In particular, Berce et al. [19] evaluated 147 children hospitalized because of ALRTI, showing that LUS detected consolidations in viral pneumonia were significantly smaller, with a median diameter of $15 \mathrm{~mm}$, compared to $20 \mathrm{~mm}$ in atypical bacterial LRTIs $(p=0.05)$ and $30 \mathrm{~mm}$ in bacterial LRTIs $(p<0.001)$. Other authors also highlighted that consolidation size or distribution can support the diagnosis of viral bronchiolitis, Influenza pneumonia and COVID-19 pneumonia [21, 28-30, 37]. These findings were comparable with our study. However, our study has evidenced more important LUS features not previously assessed. We found that air bronchograms were more common in bacteria and atypical ALRTI but, importantly, fluid bronchograms were almost exclusively described in bacterial cases. Also, complicated pleural effusions were never described in viral ALRTIs. Vertical artifacts, which gained more interest during the last year and in particular since LUS has been routinely used in COVID-19 pneumonia $[21,37-40]$, also played a significant role, since in bacterial ALRTI were mainly located in proximity of the main consolidation, while in the others were mostly diffuse and bilateral.

Considering the well-known advantages of LUS (a lowcost, easily reproducible, non-invasive tool that does not 
cause pain and damage induced by radiations), all the ultrasound findings obtained by our study can have an important impact on the daily pediatric clinical practice. LUS, particularly when used in adjunction to clinical examination by experienced operators, can support the etiological of pneumonia and offer a personalized approach to patients. If further confirmed, this approach can also support antibiotic stewardship programs.

Our study has limitations to address. The gold standard for the diagnosis of ALRTI is the chest CT scan; however, its routine use in children is not ethical in children so we did not do the CT. Therefore, the diagnosis of ALRTI for the inclusion of patients in our study was made on a clinical bases according to the available guidelines [18]. The stratification of patients according to the etiology cannot be $100 \%$ accurate, since the definitive microbiological diagnosis in non-mechanically ventilated children is difficult to establish, since bronchoalveolar lavages are invasive procedures. However, to date all studies assessing the microbiological diagnoses of ALRTI, including vaccine-probe study [41], have the same limitation that is difficult to address.

Another limitation of our study is that of not having included in the multivariable logistic regression model analysis a more specific and detailed relationship between individual etiological agents and ultrasound characteristics to make readers better understand if there were differential ultrasound identification rates of individual pathogens and therefore if there are differences in lung ultrasound findings caused by single pathogen in particular viral and atypical. Unfortunately, however, with regard to the individual viral agents (in the context of viral pneumonia) and the individual atypical agents (in the context of atypical pneumonia), we did not carry out the aforementioned correlation analyzes between the ultrasound findings and single viral or atypical pathogen because each group of single isolated pathogen (such as "RSV group" or "rhinovirus group" or "adenovirus group" or "mycoplasma group" etc.) was not sufficiently numerous to be used for a correlation analysis between a single pathogen and the associated ultrasound characteristics.

Nevertheless, we used a comprehensive assessment of each child including clinical, laboratory, imaging and microbiological data, including last generation molecular assays. On this regard, a limit is that we did not use quantitative assessments of nasopharyngeal PCR results that, according to recent studies in adult patients, have been positively associated with a diagnosis of ALRTI other than simple colonization [42].

In light of the great progress of pediatric clinical ultrasound in other sectors [43-50], our study confirms that prospects in the field of personalized medicine can also be growing for lung ultrasound.

In conclusion, our study provides a detailed analysis of LUS features able to predict the etiology ALRTI in children.
These findings may help the physicians to better manage a child with ALRTI and to offer personalized approach, from diagnosis to treatment and follow-up. However, further studies on pediatric ALRTI are necessary to confirm our findings.

Funding Open access funding provided by Università Cattolica del Sacro Cuore within the CRUI-CARE Agreement. No funds received.

Availability of data and material Available upon request.

\section{Declarations}

Conflict of interest Nothing to declare.

Ethics approval The study was approved by the Institutional Review Board and Ethic Committee of Fondazione Policlinic Universitario A. Gemelli IRCCS, Rome, Italy (prot.36173/19 ID2729).

Consent to participate Informed consent was obtained by all participants and/or their legal guardians (for children younger than 16 years). There is no identifying information or image in the article.

Consent for publication Approved.

Open Access This article is licensed under a Creative Commons Attribution 4.0 International License, which permits use, sharing, adaptation, distribution and reproduction in any medium or format, as long as you give appropriate credit to the original author(s) and the source, provide a link to the Creative Commons licence, and indicate if changes were made. The images or other third party material in this article are included in the article's Creative Commons licence, unless indicated otherwise in a credit line to the material. If material is not included in the article's Creative Commons licence and your intended use is not permitted by statutory regulation or exceeds the permitted use, you will need to obtain permission directly from the copyright holder. To view a copy of this licence, visit http://creativecommons.org/licenses/by/4.0/.

\section{References}

1. Rudan I, Tomaskovic L, Boschi-Pinto C, Campbell H, WHO Child Health Epidemiology Reference Group (2004) Global estimate of the incidence of clinical pneumonia among children under five years of age. Bull World Health Organ 82(12):895-903

2. Haq IJ, Battersby AC, Eastham K, McKean M (2017) Community acquired pneumonia in children. BMJ 356:j686

3. Gereige RS, Laufer PM (2013) Pneumonia. Pediatr Rev 34(10):438-456

4. Pereda MA, Chavez MA, Hooper-Miele CC et al (2015) Lung ultrasound for the diagnosis of pneumonia in children: a metaanalysis. Pediatrics 135(4):714-722

5. Shah SN, Bachur RG, Simel DL, Neuman MI (2017) Does this child have pneumonia?: The rational clinical examination systematic review. JAMA 318(13):1284

6. Lipshaw MJ, Eckerle M, Florin TA et al (2020) Antibiotic use and outcomes in children in the emergency department with suspected pneumonia. Pediatrics 145(4):e20193138 
7. Jain S, Williams DJ, Arnold SR et al (2015) Community-acquired pneumonia requiring hospitalization among US children. N Engl J Med 372(9):835-845

8. Jehan F, Nisar I, Kerai S et al (2020) Randomized trial of amoxicillin for pneumonia in Pakistan. N Engl J Med 383(1):24-34

9. Bradley JS, Byington CL, Shah SS et al (2011) The management of community-acquired pneumonia in infants and children older than 3 months of age: clinical practice guidelines by the Pediatric Infectious Diseases Society and the Infectious Diseases Society of America. Clin Infect Dis 53(7):e25-e76

10. Korppi M, Don M, Valent F, Canciani M (2008) The value of clinical features in differentiating between viral, pneumococcal and atypical bacterial pneumonia in children. Acta Pediatr 97:943-947

11. Flood RG, Badik J, Aronoff SC (2008) The utility of serum $\mathrm{C}$-reactive protein in differentiating bacterial from nonbacterial pneumonia in children: a meta-analysis of 1230 children. Pediatr Infect Dis J 27(2):95-99

12. Korppi M, Heiskanen-Kosma T, Leinonen M (1997) White blood cells, C-reactive protein and erythrocyte sedimentation rate in pneumococcal pneumonia in children. Eur Respir J 10(5):1125-1129

13. Berg AS, Inchley CS, Fjaerli HO, Leegaard TM, Lindbaek M, Nakstad B (2017) Clinical features and inflammatory markers in pediatric pneumonia: a prospective study. Eur J Pediatr 176(5):629-638

14. Heiskanen-Kosma T, Korppi M (2000) Serum C-reactive protein cannot differentiate bacterial and viral aetiology of communityacquired pneumonia in children in primary healthcare settings. Scand J Infect Dis 32(4):399-402

15. Korppi M (2004) Non-specific host response markers in the differentiation between pneumococcal and viral pneumonia: what is the most accurate combination? Pediatr Int 46(5):545-550

16. Stockmann C, Ampofo K, Killpack J et al (2018) Procalcitonin accurately identifies hospitalized children with low risk of bacterial community-acquired pneumonia. J Pediatric Infect Dis Soc $7(1): 46-53$

17. O'Grady KF, Torzillo PJ, Frawley K, Chang AB (2014) The radiological diagnosis of pneumonia in children. Pneumonia (Nathan) 5(Suppl 1):38-51

18. Harris M, Clark J, Coote N et al (2011) British Thoracic Society guidelines for the management of community acquired pneumonia in children: update 2011. Thorax 66(Suppl 2):ii1-ii23

19. Berce V, Tomazin M, Gorenjak M, Berce T, Lovrenčič B (2019) The usefulness of lung ultrasound for the aetiological diagnosis of community-acquired pneumonia in children. Sci Rep 9(1):17957

20. Davis T (2013) NICE guideline: feverish illness in childrenassessment and initial management in children younger than 5 years. Arch Dis Child Educ Pract Ed 98(6):232-235

21. Musolino AM, Supino MC, Buonsenso D, Roman Lung Ultrasound Study Team for Pediatric COVID-19 (ROMULUS COVID Team) et al (2020) Lung ultrasound in children with COVID-19: preliminary findings. Ultrasound Med Biol 46(8):2094-2098

22. Health NSWM of infants and children: acute management of community acquired pneumonia clinical practice guideline. Infants and children: acute management of community acquired pneumonia (nsw.gov.au)

23. Elemraid MA, Muller M, Spencer DA et al (2014) Accuracy of the interpretation of chest radiographs for the diagnosis of paediatric pneumonia. PLoS ONE 9(8):e106051

24. Musolino AM, Tomà $\mathrm{P}$, Supino $\mathrm{MC}$ et al (2019) Lung ultrasound features of children with complicated and noncomplicated community acquired pneumonia: a prospective study. Pediatr Pulmonol 54(9):1479-1486

25. Buonsenso D, Brancato F, Valentini P, Curatola A, Supino M, Musolino AM (2020) The use of lung ultrasound to monitor the antibiotic response of community-acquired pneumonia in children: a preliminary hypothesis. J Ultrasound Med 39(4):817-826

26. Lazzareschi I, Valentini P (2020) Aspiration pneumonia in children with neurological disorders: a new indication for lung ultrasound? A case series. J Ultrasound. https://doi.org/10.1007/ s40477-020-00520-4

27. Stadler JAM, Andronikou S, Zar HJ (2017) Lung ultrasound for the diagnosis of community-acquired pneumonia in children. Pediatr Radiol 47(11):1412-1419

28. Biagi C, Pierantoni L, Baldazzi M et al (2018) Lung ultrasound for the diagnosis of pneumonia in children with acute bronchiolitis. BMC Pulm Med 18(1):191

29. Tsung JW, Kessler DO, Shah VP (2012) Prospective application of clinician-performed lung ultrasonography during the 2009 H1N1 influenza A pandemic: distinguishing viral from bacterial pneumonia. Crit Ultrasound J 4(1):16

30. Buonsenso D, Musolino AM, Gatto A, Lazzareschi I, Curatola A, Valentini P (2019) Lung ultrasound in infants with bronchiolitis. BMC Pulm Med 19(1):159

31. Copetti R, Cattarossi L (2008) Ultrasound diagnosis of pneumonia in children. Radiol Med 113(2):190-198

32. Demi M, Prediletto R, Soldati G, Demi L (2020) Physical mechanisms providing clinical information from ultrasound lung images: hypotheses and early confirmations. IEEE Trans Ultrason Ferroelectr Freq Control 67(3):612-623

33. van Sloun RJG, Demi L (2020) Localizing B-lines in lung ultrasonography by weakly supervised deep learning, in-vivo results. IEEE J Biomed Health Inform 24(4):957-964

34. Soldati G, Demi M, Smargiassi A, Inchingolo R, Demi L (2019) The role of ultrasound lung artifacts in the diagnosis of respiratory diseases. Expert Rev Respir Med 13(2):163-172

35. Mongodi S, Santangelo E, De Luca D et al (2020) Quantitative lung ultrasound: time for a consensus? Chest 158(2):469-470

36. Demi L, Demi M, Prediletto R, Soldati G (2020) Real-time multifrequency ultrasound imaging for quantitative lung ultrasoundfirst clinical results. J Acoust Soc Am 148(2):998

37. Buonsenso D, Parri N, De Rose C, Valentini P, Gemelli-pediatric COVID-19 team (2021) Toward a clinically based classification of disease severity for paediatric COVID-19. Lancet Infect Dis 21(1):22

38. Parri N, Lenge M, Cantoni B et al (2020) COVID-19 in 17 italian pediatric emergency departments. Pediatrics 146(6):e20201235

39. Volpicelli G, Lamorte A, Villén T (2020) What's new in lung ultrasound during the COVID-19 pandemic. Intensive Care Med 46(7):1445-1448

40. Volpicelli G, Gargani L (2020) Sonographic signs and patterns of COVID-19 pneumonia. Ultrasound J 12(1):22

41. Obaro SK, Madhi SA (2006) Bacterial pneumonia vaccines and childhood pneumonia: are we winning, refining, or redefining? Lancet Infect Dis 6(3):150-161

42. García-Rodríguez JA, Fresnadillo Martínez MJ (2002) Dynamics of nasopharyngeal colonization by potential respiratory pathogens. J Antimicrob Chemother 50(Suppl S2):59-73

43. Brillantino C, Rossi E, Minelli R et al (2019) A rare case of renal tumor in children: clear cell sarcoma. G Chir 40(3):217-224 (PMID: 31484012)

44. Vitale V, Rossi E, Di Serafino M et al (2018) Pediatric encephalic ultrasonography: the essentials. J Ultrasound. https://doi.org/10. 1007/s40477-018-0349-7

45. Tufano A, Minelli R, Rossi E et al (2020) Inferior epigastric artery pseudoaneurysm secondary to port placement during a robotassisted laparoscopic radical cystectomy. J Ultrasound. https:// doi.org/10.1007/s40477-020-00442-1

46. Brillantino C, Rossi E, Bifano D et al (2020) An unusual onset of pediatric acute lymphoblastic leukemia. J Ultrasound. https://doi. org/10.1007/s40477-020-00461-y 
47. Minella R, Minelli R, Rossi E et al (2020) Gastroesophageal and gastric ultrasound in children: the state of the art. J Ultrasound. https://doi.org/10.1007/s40477-020-00471-w

48. Botta F, Raimondi S, Rinaldi L et al (2020) Association of a CTbased clinical and radiomics score of non-small cell lung cancer (NSCLC) with lymph node status and overall survival. Cancers 12:1432

49. Tufano A, Minelli R, Di Lascio G, Delicato G, Baffigo G, Signore S (2020) Infected kidney stone progressing to perinephric abscess and thoracic empyema. Arch Ital Urol Androl. 92(3). https://doi. org/10.4081/aiua.2020.3.203
50. Brillantino C, Rossi E, Baldari D et al (2020) Duodenal hematoma in pediatric age: a rare case report. J Ultrasound. https://doi.org/ 10.1007/s40477-020-00545-9

Publisher's Note Springer Nature remains neutral with regard to jurisdictional claims in published maps and institutional affiliations. 